\title{
Literature review and case report of bilateral extensive psoas abscess: A rare case of spondylomyelitis with infection spread to Hunter's canal with a rare causative microorganism
}

\author{
Vinodharan Nagaretnam, MD(D, Sanjiv Rampal, MBBS ${ }^{1}$, Razif Abas $^{2}$ (D), Suresh Chopra, MD ${ }^{3}$, \\ Teh Hak Lian, $\mathrm{MD}^{3}$ (i) \\ ${ }^{1}$ Department of Orthopaedic, Faculty of Medicine and Health Sciences, Universiti Putra Malaysia, Selangor, Malaysia \\ ${ }^{2}$ Department of Human Anatomy, Faculty of Medicine and Health Sciences, Universiti Putra Malaysia, Selangor, Malaysia \\ ${ }^{3}$ Department of Orthopaedic, Hospital Sultanah Bahiyah, Alor Setar, Malaysia
}

Psoas abscess is a condition where there is purulent fluid in the iliopsoas muscle compartment. This condition was initially often detected in cases of spine tuberculosis before the discovery of modern antituberculosis treatment and, later, became uncommon in developed countries after the discovery of modern antituberculosis treatment. ${ }^{[1]}$ It was often detected late during the progression of disease owing to difficulties in diagnosing this condition. However, the incidence has increased recently with improved imaging modalities such as the computed tomography (CT) scan, a helpful tool to detect the condition, particularly when clinical signs are vague. ${ }^{[2]}$ It is notable that although this

Received: January 04, 2021

Accepted: May 03, 2021

Published online: November 19, 2021

Correspondence: Sanjiv Rampal, MBBS. Department of Orthopaedic, Faculty of Medicine and Health Sciences, Universiti Putra Malaysia, 43400 Serdang, Selangor, Malaysia.

E-mail: rampalsurgery@gmail.com

Doi: $10.52312 /$ jdrs.2021.203

Citation: Nagaretnam V, Rampal S, Abas R, Chopra S, Lian TH. Literature review and case report of bilateral extensive psoas abscess: A rare case of spondylomyelitis with infection spread to Hunter's canal with a rare causative microorganism. Jt Dis Relat Surg 2021:32(3):775-778

(02021 All right reserved by the Turkish Joint Diseases Foundation

This is an open access article under the terms of the Creative Commons Attribution-NonCommercial License, which permits use, distribution and reproduction in any medium, provided the original work is properly cited and is not used for commercial purposes (http://creativecommons.org/licenses/by-nc/4.0/).

\section{ABSTRACT}

A 43-year-old male patient with underlying diabetes mellitus presented with lower abdominal pain radiating to left thigh. He was hemodynamically stable, but appeared lethargic. Both iliac fossae were tender, and crepitus appreciated along the anteromedial aspect of the left thigh. The patient initially responded well to treatment and was discharged; however, he was re-admitted several months later due to recurrent collection showing the growth of Klebsiella pneumoniae in the culture and succumbed to sepsis due to deep-seated intramuscular abscess and lung empyema. In conclusion, psoas abscess is a condition that can mimic various pathologies related to the lower abdomen and, therefore, clinicians should always have a high index of suspicion to prevent a missed diagnosis. Early detection may yield good outcomes, if managed with adequate surgical drainage and appropriate antibiotics.

Keywords: Adductor canal, Klebsiella pneumoniae, psoas abscess.

condition may progress very rapidly, it is usually confined within the iliopsoas muscle compartment and the common causative organisms are Staphylococcus aureus (S. aureus) and Mycobacterium tuberculosis. ${ }^{[3]}$

In this article, we present a rare presentation of bilateral psoas abscess with extension into the adductor canal, as well as uncommon causative organism involved.

\section{CASE REPORT}

A 43-year-old male patient with underlying diabetes mellitus and a history of admission for meningitis lesser than one year before this current condition 
presented with lower abdominal pain with radiation to the left thigh. He also complained of having fever and lethargy for the past three days and eventually unable to ambulate due to worsening pain. On physical examination, he appeared lethargic, while his vital signs were still stable. Both iliac fossae were tender, and crepitus was felt along the anteromedial aspect of the left thigh. The neurovascular status of the bilateral lower limbs was intact. Blood investigations showed lymphocytosis. A CT scan was done urgently and revealed retroperitoneal collection from the L1/L2 vertebral body and the collection was extending to bilateral iliacus muscle. However, it extended beyond the iliopsoas insertion on the left side. The CT scan images revealed also the destruction of the first and second lumbar vertebrae (Figure 1).

The patient was resuscitated adequately and, then, taken for surgical intervention on the same day as the presentation. Extensive incision and drainage were done bilaterally. Intraoperatively, more than $1,000 \mathrm{~mL}$ pus was drained, and the extensive slough tissue was debrided. The collection was found to unusually breach the deep fascia and extent into the left adductor canal (Figure 2).

During this setting, a diagnostic laparoscopy was also performed, and intra-abdominal involvement was ruled out. Specimens were sent for culture and sensitivity intraoperatively, which later revealed the growth of Klebsiella pneumoniae (K. pneumoniae). The patient was treated with intravenous amoxicillin/clavulanic acid. A repeat CT scan was done one week later and showed a residual collection. The patient was taken in for another surgical drainage, while intravenous antibiotics were continued. Subsequent repeat scan revealed only minimal residual collection, while there was no worsening of vertebral bony destruction. Although the collection was visualized to involve the L1 and L2 vertebral bodies with bony destruction, no surgical interventions were performed for the spine, as the patient did not have neurological deficits and there was no worsening of the spinal column during treatment with intravenous antibiotics.

Eventually, the patient improved clinically and was deemed fit for discharge. He was followed in the outpatient setting. However, eight months later, the patient was re-admitted for a left hip intramuscular abscess for which left hip drainage of the intramuscular abscess and arthrotomy washout was done, revealing about $40 \mathrm{~mL}$ of pus. During the same admission, the patient was also treated for lung empyema and bilateral pleural effusion by the surgical and medical team, respectively. Culture and sensitivity of specimens sent intraoperatively yielded growth of K. pneumoniae and he was treated with intravenous meropenem. Unfortunately, the patient eventually succumbed to sepsis due to
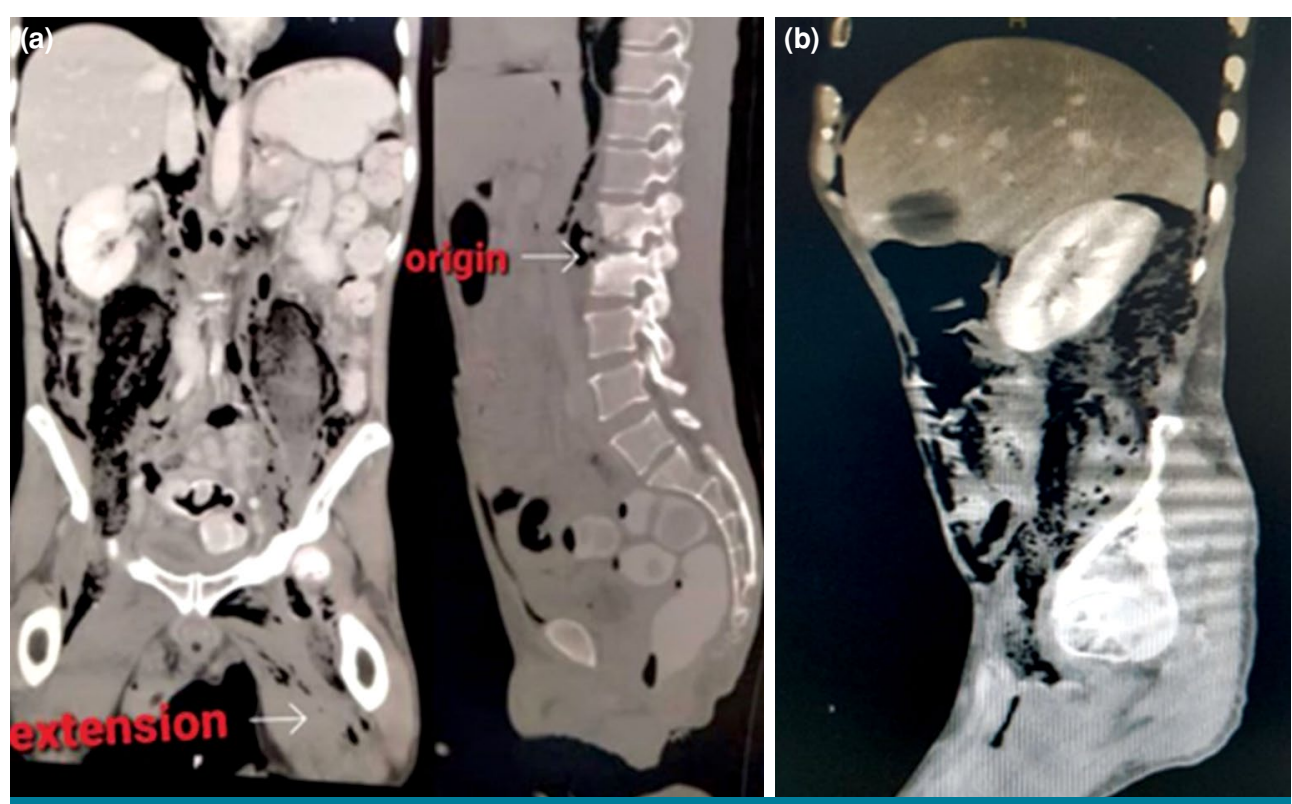

FIGURE 1. Computed tomography images of (a) coronal and (b) sagittal view showing the origin and extension of iliopsoas abscess. 

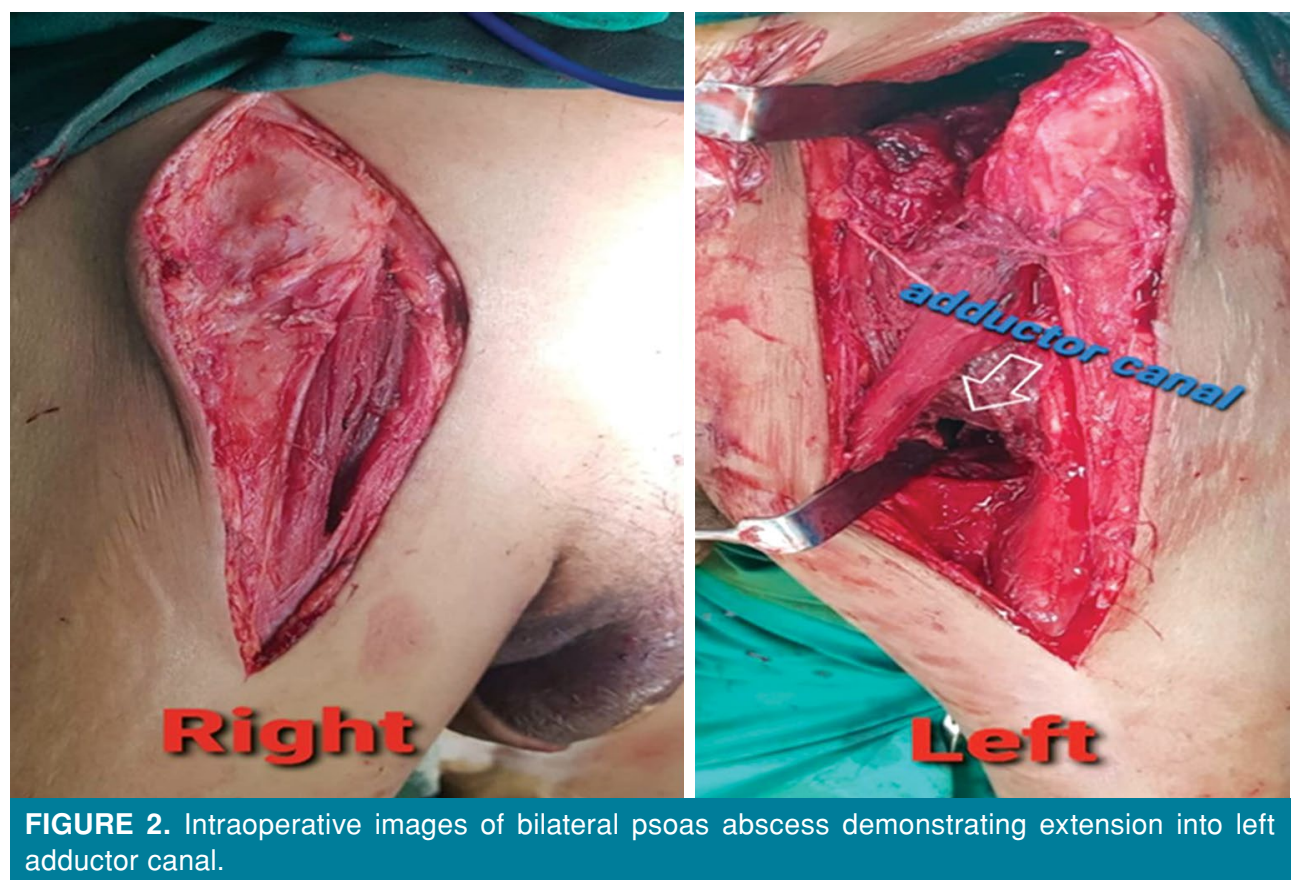

FIGURE 2. Intraoperative images of bilateral psoas abscess demonstrating extension into left adductor canal.

the deep-seated intramuscular abscess and lung empyema. A written informed consent was obtained from the patient.

\section{DISCUSSION}

This case demonstrates to us the unusual spread of psoas abscess beyond the insertion and compartment of the muscle as detected by CT and later confirmed intraoperatively during incision and drainage of the collection. Patients with psoas abscess commonly present with fever, back pain, limp and sometimes pain and lump which is confined to the groin region. ${ }^{[1]}$ Pain in the thigh region which may serve as a subtle hint for the potential atypical spread of iliopsoas abscess beyond its compartment can be sometimes taken for granted as referred pain due to innervation by L2, L3 and L4 nerve roots. ${ }^{[1]}$

It is essential to understand the anatomy of the iliopsoas muscle to be able to appreciate the complexity of this condition better. The iliopsoas compartment is located retroperitoneally which consist of iliacus, psoas major and psoas minor, as a hip flexor. ${ }^{[4]}$ The psoas muscle originates from the transverse processes of the $12^{\text {th }}$ thoracic vertebrae to the $5^{\text {th }}$ lumbar vertebrae. It courses downwardly entering the pelvic brim, passes into the inguinal ligament and runs anteriorly to the hip joint capsule which, then, inserts into the lesser trochanter of the femur after forming a tendon. The iliacus muscle joins the psoas to insert via the same tendon.

Rarely, cases of psoas abscess extend beyond the iliopsoas tendon insertion; however, in this case, it was found to extend beyond the insertion and into the adductor canal unilaterally. A case of a 43-year-old man who had a psoas abscess, which unilaterally involved the adductor muscles was described previously. ${ }^{[5]}$ Another case was a 38-year-old male patient who had a left psoas abscess with extension inferiorly to the left adductor muscles. ${ }^{[6]}$ This demonstrates to us that, during surgical drainage of the abscess, a thorough exploration of surrounding structures with adequate care is recommended to avoid incomplete drainage of the purulent fluid in rare cases where it extends beyond the usual compartment of the iliopsoas muscle which would eventually lead to morbidity and mortality.

Although the quadriceps muscle is the commonest site of abscess extension followed by the glutei, ${ }^{[7]}$ a case report demonstrated the involvement of the entire compartment of thigh adductor spread from primary psoas abscess. ${ }^{[5]}$ Although this patient had no comorbidities, he presented with a copious amount of pus from the psoas muscle. However, no further extension was observed. This report proved that a large amount of abscess was susceptible for its additional 
route. Additionally, it was well mentioned that the abscess might destroy the part of the psoas fascia or muscle hence extend further inferiorly to the femoral triangle via inguinal ligament involvement and subsequent communication to the adductor canal. ${ }^{[8,9]}$ However, the presence of the vastoadductor membrane surrounds the adductor canal prevent a further spread superficially. ${ }^{[10]}$

Similarly, another case study proved that a high amount of psoas abscess spread up to the thigh and adductor muscles. After four days of antibiotic therapy, the patient demonstrated sartorius muscle necrosis and muscle necrosectomy was performed. However, this patient had a concurrent perforation of appendiceal and adductor areas. ${ }^{[1]]}$ Interestingly, an isolated adductor brevis pyomyositis was documented in a patient with a psoas abscess presenting with low back left thigh and groin pain whose urogenital and abdominal systemic symptoms were unremarkable. ${ }^{[12]}$

It is also worth noting the uncommon causative organism, which was isolated in this case of psoas abscess, being K. pneumoniae. However, it has been widely demonstrated that $S$. aureus is the common causative organism in most cases of psoas abscess. It was also shown that a 78-year-old male patient who recovered from a liver abscess immediately developed bilateral gas-forming pyogenic psoas abscess caused by K. pneumoniae. ${ }^{[13]}$ This occurrence of the rare causative organism in this condition can serve as a guide to us to be more vigilant with our choices of antibiotics in suspected or confirmed cases of psoas abscess. ${ }^{[14]}$ In conclusion, psoas abscess is a condition that can mimic various pathologies related to the lower abdomen and, therefore, clinicians should always have a high index of suspicion to prevent a missed diagnosis of this condition which may prove to be fatal. We should always attempt to identify the actual extent of psoas abscess, as it can involve unusual adjacent areas such as the adductor canal. Our case also demonstrates that other organisms such as $K$. pneumoniae may be implicated and, thus, prompt and appropriate antibiotic coverage should be initiated. We would also like to emphasize that a postoperative CT scan should be performed as infection may persist despite surgery. Although this condition is associated with significant morbidity and mortality, it can still have good outcomes, if diagnosed early and managed adequately with surgical intervention and antibiotic coverage.

\section{Acknowledgement}

We would like to acknowledge the Head Department of Orthopaedic and Traumatology for all the support and vision in the publication of this paper.

\section{Declaration of conflicting interests}

The authors declared no conflicts of interest with respect to the authorship and/or publication of this article.

\section{Funding}

The authors received no financial support for the research and/or authorship of this article.

\section{REFERENCES}

1. Mallick IH, Thoufeeq MH, Rajendran TP. Iliopsoas abscesses. Postgrad Med J 2004;80:459-62.

2. Zissin R, Gayer G, Kots E, Werner M, Shapiro-Feinberg M, Hertz M. Iliopsoas abscess: A report of 24 patients diagnosed by CT. Abdom Imaging 2001;26:533-9.

3. Wong OF, Ho PL, Lam SK. Retrospective review of clinical presentations, microbiology, and outcomes of patients with psoas abscess. Hong Kong Med J 2013;19:416-23.

4. Spelman D. Psoas abscess. UpToDate MA: Wellesley: 2009. Available at: http://somepomed.org/articulos/contents/ mobipreview.htm?23/62/24559?source=related_link

5. Zhou Z, Song Y, Cai Q, Zeng J. Primary psoas abscess extending to thigh adductors: Case report. BMC Musculoskelet Disord 2010;11:176.

6. Xu BY, Vasanwala FF, Low SG. A case report of an atypical presentation of pyogenic iliopsoas abscess. BMC Infect Dis 2019;19:58.

7. Chiedozi LC. Pyomyositis. Review of 205 cases in 112 patients. Am J Surg 1979;137:255-9.

8. Adelekan M, Taiwo S, Onile BA. A review of psoas abscess. Afr J Clin Exp Microbiol 2004;5:55-63.

9. Panchamia JK, Niesen AD, Amundson AW. Adductor canal versus femoral triangle: Let us all get on the same page. Anesth Analg 2018;127:e50.

10. Johnston DF, Black ND, Cowden R, Turbitt L, Taylor S. Spread of dye injectate in the distal femoral triangle versus the distal adductor canal: A cadaveric study. Reg Anesth Pain Med 2019;44:39-45.

11. Petrovic I, Pecin I, Prutki M, Augustin G, Nedic A, Gojevic A, et al. Thigh abscess as an extension of psoas abscess: The first manifestation of perforated appendiceal adenocarcinoma: Case report. Wien Klin Wochenschr 2015;127:645-8.

12. Devetag Chalaupka F. Acute iliopsoas and adductor brevis abscesses presenting with proximal leg muscle weakness. Neurol Sci 2006;27:125-8.

13. Liao PY, Chiang WC, Chen SY, Su CP, Wang JT, Hsueh PR. Rapidly fatal gas-forming pyogenic psoas abscess caused by Klebsiella pneumoniae. Clin Infect Dis 2007;44:1253-5.

14. Atik OŞ. Is there something new and interesting in my article? Eklem Hastalik Cerrahisi 2019;30:69. 